\title{
Life-Threatening Autoimmune Hemolytic Anemia Treated with Manual Whole Blood Exchange with Rapid Clinical Improvement
}

\author{
Laura Cooling $^{1 *}$, Grace Boxer ${ }^{2}$ and Richard Simon ${ }^{2}$ \\ ${ }^{1}$ Department of Pathology, University of Michigan, Ann Arbor, MI, USA \\ ${ }^{2}$ Department of Internal Medicine, University of Michigan, Ann Arbor, MI, USA
}

\begin{abstract}
Severe autoimmune hemolytic anemia, with high titer panagglutinins, can present with severe intra-and extravascular hemolysis, complicated by renal failure, volume overload, hypertriglyceridemia, pancreatitis and multiorgan failure. We present a case of life-threatening warm autoimmune hemolytic anemia in a 19 year old male, refractory to steroids and splenectomy, with ongoing severe hemolysis, in vivo and in vitro autoagglutination, worsening hypoxia, methemoglobinemia, renal insufficiency, and hypertriglyceridemia. The patient underwent a single manual whole blood exchange with reconstituted whole blood ( $50 \%$ final hematocrit). The post-exchange hemoglobin was $7.7 \mathrm{gm} / \mathrm{dL}$, with complete resolution of RBC autoagglutination, increased platelet count (20\% ) and marked decreases in plasma free hemoglobin (43\%), methemoglobin (9\%), percent $\mathrm{FiO}_{2}(40 \%)$, creatinine $(30 \%)$, triglycerides $(66 \%)$, bilirubin (56\%) and autoantibody titer (1000 to 256$)$. The patient was extubated shortly after WBEx with minimal hemolysis and RBC transfusion support over the next 2 weeks.
\end{abstract}

Keywords: Blood exchange, Autoimmune hemolytic anemia

Abbreviations: AIHA: Autoimmune Hemolytic Anemia; DAT: Direct Antiglobulin Test; Hgb: Hemoglobin; IAT: Indirect Antiglobulin Test; 2-ME: 2-Mercaptoethanol; Prbc: Packed RBC Units; SCID: Severe Combined Immunodeficiency Disorder; WAIHA: Warm Autoimmune Hemolytic Anemia; WB: Whole Blood; Wbex: Whole Blood Exchange

\section{Introduction}

Autoimmune hemolytic anemia (AIHA) is a relatively rare disorder with an incidence of 1-3 per 100,000 [1]. Warm AIHA (WAIHA) is typically due to $\operatorname{IgG}$ autoantibodies with optimal reactivity at $37^{\circ} \mathrm{C}$ and account for the vast majority of AIHA cases (80-90\%) $[1,2]$. Clinical presentation can range from an asymptomatic, compensated hemolytic anemia to acute life-threatening hemolysis. Hemolysis is typically extravascular; however, complement-mediated intravascular hemolysis can be seen at high levels of RBC bound antibody (>1000 molecules IgG), in rare IgM WAIHA and in mixed AIHA characterized by IgM and IgG autoantibodies [2-5]. Although death from WAIHA is considered rare, mortality rates of $4-11 \%$ have been reported in children [6,7]. Mortality is particularly high in mixed and WAIHA due to IgM autoantibodies possessing high thermal amplitudes [3-5].

The standard treatment for WAIHA is high dose steroids, which are effective in $70-80 \%$ of patient within 2 weeks of starting therapy $[1,2]$. Steriod-refractoriness is observed in approximately $20 \%$ of patients, who are typically treated by either splenectomy and/or a trial of rituximab. Alternative salvage therapies for resistant WAIHA, as single agents or in combination, are danazol, IVIG, alemtuzumab, cyclophosphamide, azathioprine, cyclosporine, mycophenolate mofetil, and vincristine-loaded platelets [1,8-10]. Plasmapheresis has also been tried in severe AIHA as adjunct therapy, usually in combination with multi-agent immunosuppressive regimens [11-13].

There are a handful of reports utilizing whole blood exchange (WBEx) in severe AIHA. In general, WBEx has been used to avoid volume overload in patients requiring massive RBC transfusion support or to treat in vivo hemagglutination [4,5,14-16]. We report the use of manual WBEx in a young man with life-threatening hemolysis, RBC autoagglutination, respiratory failure, methemoglobinemia, impending acute renal failure and hemolysis-associated hypertriglyceridemia.
Following a single WBEx, the patient defervesced with rapid improvement in oxygenation, renal function and hemolysis.

\section{Materials and Methods}

\section{Serology}

Samples for patient testing were collected in EDTA per routine. Spontaneous agglutination was resolved by washing patient's red cells with prewarmed saline at $37^{\circ} \mathrm{C}$ four times [3]. ABO and $\mathrm{Rh}(\mathrm{D}, \mathrm{C}, \mathrm{E})$ $\mathrm{RBC}$ typing were performed with commercial reagents (Ortho Clinical Diagnostics, Raritan, NJ). An extended RBC genotype was performed by the University of Michigan Molecular Pathology Laboratory using the Red Cell EZ type reagents (Hologics/GenProbe, San Diego, CA). The patient's predicted RBC phenotype was rr, kk, Jk $(a-b+), F y(a+b+)$, MNss.

Direct antiglobulin testing (DAT) was performed by tube method using commercial anti-human IgG, anti-human C3 and polyspecific (IgG, C3) reagents: 6\% albumin was always included as an inert control. IgG isotype analysis was not performed. RBC eluates were prepared with EDTA-glycine-acid (EGA ${ }^{\mathrm{TM}}$ kit, Immucor/Gamma, Norcross, GA). Eluates were tested against untreated and ficin-treated RBC using polyethylene glycol enhancement (GammaPeG ${ }^{\mathrm{TM}}$, Immucor/Gamma).

Plasma reactivity was examined at immediate spin, room temperature; 60 minute, $37^{\circ} \mathrm{C}$ incubation and the IAT against

*Corresponding author: Laura Cooling, Associate Professor, Pathology University of Michigan Hospitals, 2F225 UH Blood Bank, Box 0054, 1500 East Medical Center Drive, Ann Arbor, MI 48109-0054, USA, Tel: 734-936-0695; Fax: 734-936-6855; E-mail: Icooling@med.umich.edu

Received July 25, 2013; Accepted October 08, 2013; Published October 12 2013

Citation: Cooling L, Boxer G, Simon R (2013) Life-Threatening Autoimmune Hemolytic Anemia Treated with Manual Whole Blood Exchange with Rapid Clinical Improvement. J Blood Disorders Transf 4: 163. doi: 10.4172/2155 9864.1000163

Copyright: (c) 2013 Cooling L, et al. This is an open-access article distributed under the terms of the Creative Commons Attribution License, which permits unrestricted use, distribution, and reproduction in any medium, provided the original author and source are credited. 
unmodified and ficin-treated RBC [17]. Indirect antiglobulin testing (IAT) was performed by tube and gel method using anti-IgG and anti-polyspecific reagents. To remove autoantibody, plasma was alloadsorbed ( $\mathrm{x} 4$ ) against group $\mathrm{O}$, rr, Jk (a-) RBC treated with ZZAP $[17,18]$. To exclude IgM autoantibody, patient plasma was incubated with PBS or 2-mercaptoethanol (2-ME) for 2 hours at $37^{\circ} \mathrm{C}$, followed by overnight dialysis as described [17]. Autoantibody titration was performed by the IAT tube method $\left(60^{\prime}, 37^{\circ} \mathrm{C}\right.$, anti-IgG) using two-fold dilutions of plasma diluted in normal saline and tested against group $\mathrm{O}$, rr, unmodified RBC. The autoantibody titer was the last dilution showing $1+$ reactivity [17]. The autoantibody score was calculated as described by Marsh [19].

\section{Whole blood exchange}

Reconstituted whole blood (WB) was prepared from group A plasma and pre-storage leukoreduced, group $\mathrm{A}, \mathrm{Rh}$-negative packed red blood cells ( $\mathrm{pRBC}$ ) in Adsol anticoagulant. PRBC units were less than 15 days of age and matched for Rh and $\mathrm{K}$ antigens (C-, E-, K-). PRBC were centrifuged and resuspended in plasma to a $50 \%$ final hematocrit (Hct) [20]. Because of its short outdate [21], WB was prepared and dispensed one unit at a time. WB units were crossmatched against unadsorbed patient plasma and labeled 'crossmatch-incompatible'. For WBEx, a right internal jugular cordis central line was placed for venous access. WB was removed with $50 \mathrm{~mL}$ syringes over a period of 10-15 minutes, followed by infusion of WB.

\section{Case Description}

The patient was a morbidly obese (weight $121 \mathrm{~kg}$, BMI 40.4), 19 year old white male who presented to his local emergency room with a 5 day history of fatigue, malaise, anorexia, right flank pain, dark urine and new nausea and vomiting. His history was unremarkable except for intermittent headaches during the last 2 months. He was not taking prescription medication and had no prior significant past medical history. Laboratory studies showed anemia and hyperbilirubinemia. The patient was subsequently transferred to a larger facility for further evaluation and treatment.

On admission to the second facility, the patient was febrile and jaundiced, with a hemoglobin (Hgb) $7.7 \mathrm{gm} / \mathrm{dL}$, an elevated LDH (1625 $\mathrm{IU} / \mathrm{L})$, low haptoglobin $(<10 \mathrm{mg} / \mathrm{dL})$ and indirect hyperbilirubinemia (total bilirubin=10.4 mg/dl, indirect bilirubin=10 mg/dL). The patient typed as group $\mathrm{A}$, Rh-negative with a positive antibody screen due to a strong (4+) panagglutinin. His DAT was reportedly positive with both anti-IgG and anti-C3. A CT scan showed splenomegaly and bilateral pulmonary lower lobe opacities with consolidation. No lymphadenopathy or masses were noted by CT or physical exam. The patient was admitted and received 4 units of group A pRBC with no increase in Hgb. Within 12 hours of admission, the patient was transferred to the ICU with tachypnea $(\mathrm{RR}=28)$, tachycardia $(\mathrm{HR}=129)$, hypotension $(\mathrm{SBP}=80)$, hypoxia $\left(\right.$ Percent $\mathrm{O}_{2}$ saturation $=80 \%$ on $\left.6 \mathrm{~L} \mathrm{O}_{2}\right)$ and new nonspecific ST changes by EKG. The patient was intubated and transferred to a university medical center the morning after admission.

On arrival to the university hospital, the patient was febrile $\left(\mathrm{T}=39.1^{\circ} \mathrm{C}\right)$, tachycardic $(\mathrm{HR}=112 \mathrm{bpm})$, normotensive $(\mathrm{BP}=140 / 55)$, intubated $\left(\% \mathrm{FiO}_{2}=40 \%, \mathrm{O}_{2}\right.$ saturation $\left.=95-99 \%\right)$ with jaundice and palpable splenomegaly. He had worsening anemia $(\mathrm{Hgb}=5.7 \mathrm{gm} / \mathrm{L})$ with elevated reticulocytes $(0.25 \%)$ but normal $\mathrm{RBC}$ indices, platelet $\left(164 \times 10^{9} / \mathrm{L}\right)$ and $\mathrm{WBC}$ counts $\left(11.7 \times 10^{9} / \mathrm{L}\right)$. A peripheral blood smear showed some rouleaux but no increase in spherocytes or schistocytes. A WBC differential showed an absolute and relative neutrophilia
(79\%) with toxic granulation and dohle bodies. Chemistry studies showed absent haptoglobin $(<10 \mathrm{mg} / \mathrm{dL})$; elevated plasma free $\mathrm{Hgb}$ $(214 \mathrm{mg} / \mathrm{dL}), \mathrm{LDH}(2686 \mathrm{IU} / \mathrm{L})$, AST $(675 \mathrm{IU} / \mathrm{L})$, indirect bilirubin (total bilirubin $=11.6 \mathrm{mg} / \mathrm{dL}$, indirect $=10.2 \mathrm{mg} / \mathrm{dL}$ [ $88 \%$ total]), and hemoglobinuria. (79\%) with toxic granulation and dohle bodies. Chemistry studies showed absent haptoglobin $(<10 \mathrm{mg} / \mathrm{dL})$; elevated plasma free Hgb (214 mg/dL), LDH (2686 IU/L), AST (675 IU/L), indirect bilirubin (total bilirubin $=11.6 \mathrm{mg} / \mathrm{dL}$, indirect $=10.2 \mathrm{mg} / \mathrm{dL}$ [88\% total]), and Coagulation studies showed a normal PT, aPTT, $\mathrm{d}$-dimer and elevated fibrinogen $(550 \mathrm{mg} / \mathrm{dL})$. The patient's admission renal function showed a prerenal azotemia with elevated BUN (49 mg/ $\mathrm{dL})$, slightly elevated creatinine $(1.5 \mathrm{mg} / \mathrm{dL})$ and abundant granular casts (26-50/hpf) on urine microscopy. An admission lipid panel showed mildly elevated triglycerides ( $248 \mathrm{mg} / \mathrm{dL}$; cholesterol, $177 \mathrm{mg} /$ dL). An abdominal ultrasound showed splenomegaly. A chest X-Ray showed no acute disease.

Diagnostic tests were unrevealing. The patient was negative for cryoglobulins, cold agglutinins, ANA, ANCA, MPO, PR3, and antiphosholipid antibodies. Serum IgG, IgM, IgA and C3 levels were normal; however, the $\mathrm{C} 4$ was low $(0.2 \mathrm{mg} / \mathrm{dL})$. A serum electrophoresis showed an acute phase pattern only: No C-reactive protein or ESR was performed. Several sets of blood, urine and sputum cultures were negative. Serology and PCR studies showed no evidence of acute infection with CMV, EBV, HIV, HBV or HCV. Thrombotic thrombocytopenia purpura (ADAMST13 activity=48\%) and paroxysmal nocturnal hemoglobinuria (no detectable CD14-, FLAERmonocytes) were excluded. The patient did have an elevated urine copper $(1035 \mu \mathrm{g} / 24$ hours; normal range $<55 \mu \mathrm{g})$ suggestive of Wilson's disease; however, the patient had repeatedly normal ceruloplasmin

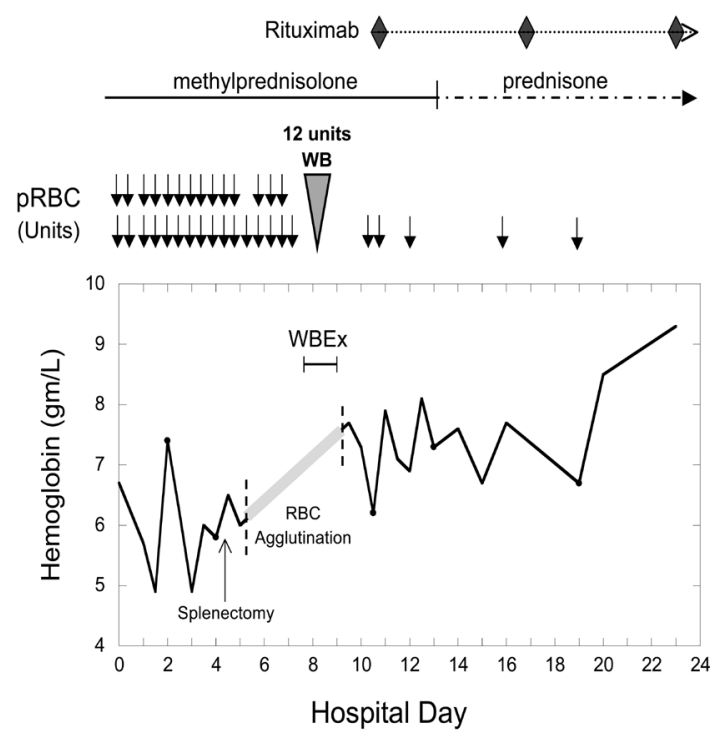

Figure 1: Hemoglobin levels (-) and RBC transfusion (arrows) during the first 3 weeks of hospitalization. No reportable hemoglobin levels (gray line) could be obtained between day 5 and day 8 due to severe RBC autoagglutination that interfered with laboratory testing. Patient received manual whole blood exchange (WBEx) with 12 units reconstituted WB (triangle) starting late on day 7 through day 8 Immunosuppression included methprednisolone (Day 1-13), prednisone (starting day 14), and 4 weekly doses of rituximab starting on day 10 (gray diamonds). 


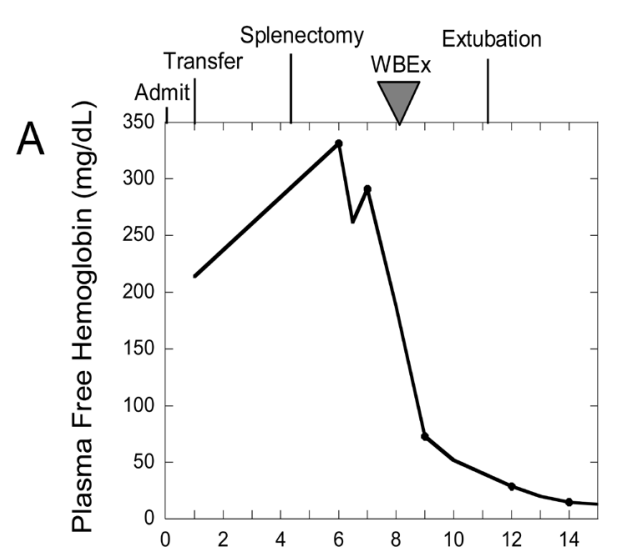

$\mathrm{B}$

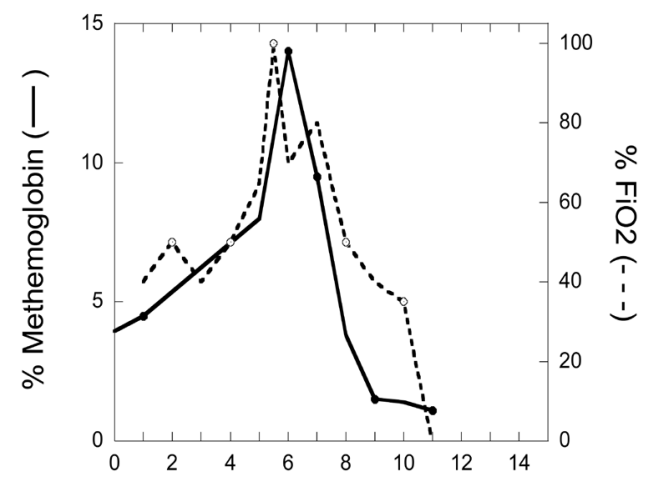

C

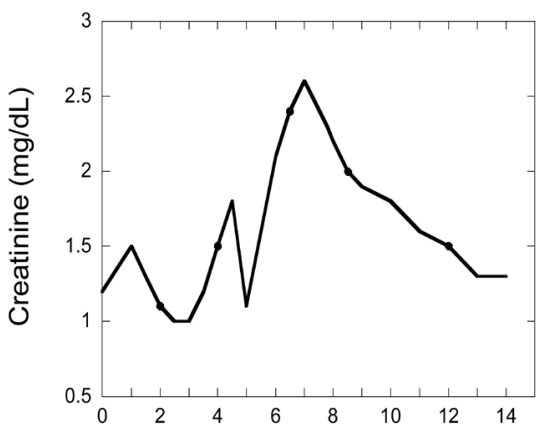

D

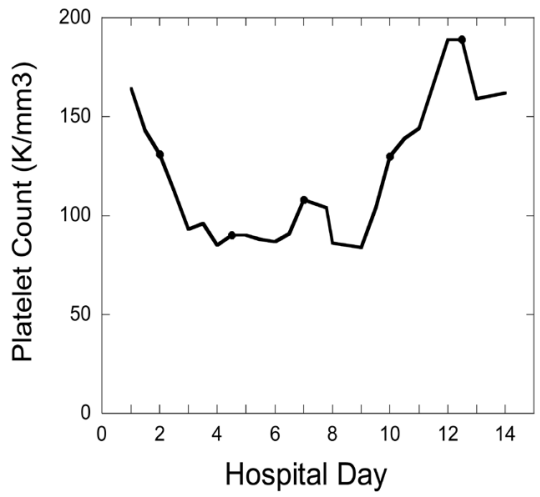

Figure 2: Correlation between plasma free hemoglobin (A) and percent (\%) methemoglobin and $\mathrm{FiO} 2(\mathrm{~B})$, serum creatinine $(\mathrm{C})$ and platelet count $(\mathrm{D})$.

levels (x2), no ATP7B gene mutations, and no Kayser-Fleisher rings on slit lamp exam.

Blood bank testing was complicated by the presence of a strong autoantibody with both RBC autoagglutination and spontaneous agglutination [3], even after washing patient RBC eight times with normal saline. Agglutination was resolved after repeatedly washing RBC with warm saline $[3,17]$. The DAT was positive with polyspecific $(4+)$ and IgG (4+) but not complement. His plasma contained a strong panreactive antibody with anti-IgG at $37^{\circ} \mathrm{C}$ by gel and tube methods. Reactivity was enhanced with ficin-treated RBC, but not by incubation at cooler temperatures. An eluate was positive with all cells tested in the IAT. Plasma reactivity was not affected by treatment with 2-ME. No alloantibodies were identified after testing adsorbed plasma.

Upon admission, the patient was treated with methylprednisolone (6 mg/kg), pRBC transfusions, folic acid, antibiotics (azithromycin, vancomycin) and hydration to prevent pigment nephropathy. Over the course of 3 days, the patient was transfused with 14 units pRBC with no sustained increase in Hgb (Figure 1). On day 4, the patient underwent an open splenectomy with minimal intraoperative blood use (1 pRBC). The spleen was enlarged, measuring $15 \mathrm{~cm}$ and weighing $730 \mathrm{gm}$. White pulp atrophy consistent with steroids, extramedullary hematopoiesis and florid hemophagocytosis were noted on histology.

Following splenectomy, the patient had continuing hemolysis accompanied by worsening spontaneous hemagglutination $\left(37^{\circ} \mathrm{C}\right)$ and RBC microaggregates on peripheral blood smears (Figure 1). In addition, the patient's DAT was now positive for complement $(\mathrm{IgG}=4+$, $\mathrm{C} 3=2+$ ). The patient's clinical condition continued to decline with increasing renal insufficiency (Figure $2 \mathrm{C}$, peak creatinine $=2.6 \mathrm{mg} /$ $\mathrm{dL}, \mathrm{BUN}=100-108)$, fever $\left(\operatorname{Tmax}=40^{\circ} \mathrm{C}\right)$ and new rhabdomyolysis (creatinine kinase $=3490 \mathrm{IU} / \mathrm{dL}$ ). He developed increasing oxygenation requirements $\left(\mathrm{FiO}_{2}=80-100 \%, \mathrm{O}_{2}\right.$ saturation=80-90\%), concurrent with rising methemoglobinemia (peak 14.7\%), cyanosis and lactate (peak lactate $=7.2 \mathrm{mEq} / \mathrm{L}$ ). There was a ten-fold increase in triglycerides ( 248 to $2653 \mathrm{mg} / \mathrm{dL}$; cholesterol=177 $\mathrm{mg} / \mathrm{dL}$ ), felt to be secondary to massive hemolysis and systemic inflammation [22]. A serum amylase and lipase were not performed. The patient was placed on esmolol for new hypertension (BP 200s/90-107). A trial of rituximab on day 6 was suspended after the patient had a reaction to the test dose (100 mg).

On day 7 , the hospital transfusion medicine service was consulted regarding possible plasmapheresis as prophylaxis against acute renal failure and pancreatitis by pigment nephropathy and hypertriglyceridemia, respectively [23-25]. In addition, it was hoped that plasmapheresis might slow the rate of hemolysis. After review, it was felt that the patient was not sufficiently stable to undergo apheresis. Furthermore, the severity of hemagglutination at $37^{\circ} \mathrm{C}$ raised technical concerns regarding the ability to perform the procedure. Specifically, there was a significant risk of RBC aggregation, procedure-related hemolysis, and circuit occlusion with circuit loss by exposure of patient blood to the centrifugal shear forces and cooler temperatures within the extracorporeal circuit.

Instead of plasmapheresis, it was decided to proceed with a manual WBEx using reconstituted WB. Unlike apheresis, manual WBEx had minimal clinical or procedural risks, while providing $\mathrm{RBC}$ transfusion support. In addition, WBEx could remove triglycerides and plasma free $\mathrm{Hgb}$ as well as methemoglobin and circulating RBC microagglutinates. Given the severity of the patient's hemolysis, we opted to reconstitute pRBC to a standard final Hct of $50 \%$ used in neonatal WBEx. His estimated total blood volume, corrected for BMI, was $4800 \mathrm{~mL}$. The patient received $12 \mathrm{WB}$ units $(4320 \mathrm{~mL}, 89 \%$ total blood volume) over 20 hours with no adverse events. His post-procedure $\mathrm{Hgb}=7.7 \mathrm{gm} / \mathrm{dL}$ and was accompanied by a resolution of RBC autoagglutination (Figure $3 \mathrm{~A}$ and $3 \mathrm{~B}$ ). WBEx successfully decreased methemoglobin (Figure $2 \mathrm{~B}$ ), 
Citation: Cooling L, Boxer G, Simon R (2013) Life-Threatening Autoimmune Hemolytic Anemia Treated with Manual Whole Blood Exchange with Rapid Clinical Improvement. J Blood Disorders Transf 4: 163. doi: 10.4172/2155-9864.1000163

Page 4 of 6

plasma free Hgb (43\%, Figure 1A), triglycerides (66\%, 2653 to $903 \mathrm{mg} /$ $\mathrm{dL})$, and total bilirubin $(56 \%, 13.6$ to $5.9 \mathrm{mg} / \mathrm{dL})$.

Following WBEx, the patient became afebrile. There was a significant improvement in hemolysis as evidenced by decreased $\mathrm{pRBC}$ transfusion frequency (Figure 1) and falling plasma free $\mathrm{Hgb}$ with clearance of serum (Figure 1A and 3C). The resolution in hemolysis was accompanied by improvements in renal function and platelet count (Figure $2 \mathrm{C}$ and 2D). The patient's percent $\mathrm{FiO}_{2}$ requirements dropped rapidly with extubation early on Day 11 (Figure 2B). Parallel testing of his pre- and post-WBEx samples showed a significant decrease in autoantibody titer (1000 to 256) and Marsh score (108 to 82).

The patient was successfully rechallenged with rituximab on day 10 followed by 3 additional weekly doses. He was transitioned from methylprednisolone to oral prednisone on day 14. Following WBEx, the patient required only 5 more pRBC transfusions over the next 11 days, with his last transfusion on day 19 (Figure 1). His autoantibody levels continued to fall over the next two weeks (IAT $=2+$, neat plasma; day 25). He was discharged from the hospital on day 43. Testing 18 months later showed a negative DAT and antibody screen.

\section{Discussion}

With rare exceptions, WAIHA is due to IgG autoantibodies, with $50-75 \%$ of cases also positive for complement [2,7]. Patients can present with a severe hemolytic anemia requiring aggressive medical treatment and transfusion support. Due to their severe anemia, these patients are at risk for volume overload and pulmonary edema from frequent transfusions, an expanded plasma volume, and hydration to prevent pigment nephropathy. Additional complications in these patients include renal failure, disseminated intravascular coagulation and multiorgan failure from massive, ongoing intravascular release of free Hgb and RBC stroma [1,25]. Death in WAIHA is usually the result of cardiovascular collapse, pulmonary emboli or infection $[1,4,5]$.

We present a case of life-threatening, refractory WAIHA that was successfully treated with a single manual WBEx with rapid and dramatic clinical improvement. Manual WBEx was chosen over plasmapheresis in our patient since it presented minimal risk, provided $\mathrm{RBC}$ transfusion support, and permitted concurrent plasma and RBC exchange. A single manual WBEx led to acceptable decreases in plasma free Hgb, triglycerides, and bilirubin. WBEx was also highly effective

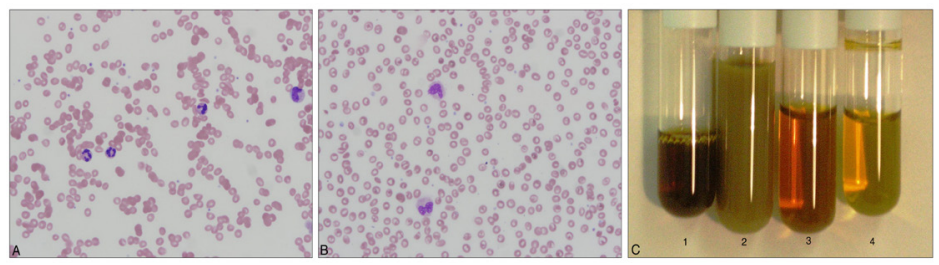

Figure 3: Peripheral blood smear pre-WBEx (A) and post-WBEx (B) showing clearing of RBC autoagglutination.

C) Serum samples from admission (1), pre-WBEx (2), 24 hours post-WBEx (3) and 48 hours post-WBEx (4).

\begin{tabular}{|c|c|c|c|c|c|c|c|}
\hline Diagnosis & Age*/Sex & DAT & Procedure & Replacement Fluid & Clinical Response & Outcome & Reference \\
\hline $\begin{array}{c}\text { WAIHA } \\
\text { Post-infectious } †\end{array}$ & $40 / \mathrm{M}$ & $\begin{array}{l}\lg { }^{+}+ \\
\text {C3+ }\end{array}$ & $\begin{array}{c}\text { Phlebotomy }(900 \mathrm{~mL}) \\
+ \\
\text { Plasmapheresis }(2400 \\
\mathrm{mL})\end{array}$ & $\begin{array}{c}2 \mathrm{pRBC} \\
700 \mathrm{~mL} \mathrm{WB}, \\
450 \mathrm{~mL} \text { plasma } \\
500 \mathrm{~mL} \text { Haemaccel }\end{array}$ & $\begin{array}{l}\text { Rapid Improvement } \\
\text { Afebrile } \\
\text { Stable Hgb } \\
\text { Decreased hemolysis } \\
\text { Improved renal function } \\
\text { Decreased DAT }\end{array}$ & Survived & Garelli [14] \\
\hline $\begin{array}{c}\text { Refractory CLL } \\
\text { WAIHA } \\
\text { Volume overload }\end{array}$ & $54 / F$ & $\lg \mathrm{lg}+$ & $\begin{array}{l}\text { 2L WBEx using } \\
\text { Infusion pumps }\end{array}$ & $\begin{array}{l}\text { Reconstituted RBC (saline, } \\
\text { albumin, plasmalyte,plasma) }\end{array}$ & $\begin{array}{l}\text { Improvement } \\
\text { Decreased DAT }\end{array}$ & Survived & Becker [15] \\
\hline WAIHA & 19/M & $\lg \mathrm{G}+ \pm \mathrm{C} 3+$ & $\begin{array}{l}\text { WBEx } \\
(4300 \mathrm{~mL})\end{array}$ & WB & $\begin{array}{l}\text { Rapid improvement } \\
\text { Decreased hemolysis } \\
\text { Improved oxygenation } \\
\text { Decreased DAT }\end{array}$ & Survived & This case \\
\hline $\begin{array}{c}\text { SCID } \\
\text { Haploidential-BMT } \\
\text { IgM-WAIHA }\end{array}$ & $0.9 / F$ & $\mathrm{C} 3+$ & WBEx & WB & $\begin{array}{c}\text { None } \\
\text { Ongoing hemolysis } \\
\text { Autoagglutination } \\
\text { CVA } \times 2\end{array}$ & Deceased & $\begin{array}{l}\text { Friedmann } \\
{[4]}\end{array}$ \\
\hline $\begin{array}{l}\text { Evan's Syndrome } \\
\text { IgM-WAIHA }\end{array}$ & 9/F & $\mathrm{C} 3+$ & WBEx & WB & $\begin{array}{c}\text { None } \\
\text { Ongoing hemolysis } \\
\text { Autoagglutination } \\
\text { Multiple CVA } \\
\text { Pulmonary emboli } \\
\text { Renal failure } \\
\text { Ischemic gangrene }\end{array}$ & Deceased & $\begin{array}{c}\text { Nowak- } \\
\text { Wegrzyn[5] }\end{array}$ \\
\hline $\begin{array}{c}\text { Mycoplasma } \\
\text { Anti-Pr cold AlHA }\end{array}$ & $41 / \mathrm{M}$ & $\mathrm{C} 3+$ & WBEx & WB & None & Deceased & $\begin{array}{c}\text { Schonitzer } \\
{[16]}\end{array}$ \\
\hline
\end{tabular}

BMT: Bone Marrow Transplantation; CVA: CerebroVascular Accident; CLL: Chronic Lymphocytic Leukemia; DAT: Direct Antiglobulin Test; SCID: Severe Combined Immuno Deficiency.

${ }^{*}$ Age in years

†Upper respiratory tract infection with fever in the last month.

$\ddagger$ Presumed post-infectious

Table 1: Use of whole blood exchange in AlHA 
in reducing methemoglobin, with parallel improvement in oxygenation and ventilator settings. Methemoglobin levels as low as $8-10 \%$ is associated with low oxygenation saturation and hypoxia due to a loss of reversible $\mathrm{O}_{2}$ binding and left-shift in the $\mathrm{O}_{2}$ dissociation curve [26-29].

Most surprising, however, was the resolution of RBC autoagglutination, $\mathrm{C} 3$ positivity and hemolysis shortly following WBEx. The patient showed continuing clearance of free Hgb for several days after the procedure (Figure 1 and $3 \mathrm{C}$ ). More importantly, his transfusion requirements dropped from 31 units pre-WBEx to only $5 \mathrm{RBC}$ units post-WBEx. Two potential and complementary mechanisms for these findings are in vivo alloadsorption of free autoantibody and removal of heavily-coated, sensitized RBC. During the course of WBEx, the patient slowly received approximately $2100 \mathrm{~mL}$ pRBC, which provided time and substrate for autoantibody adsorption. As a result, WBEx may have lowered the amount of RBC-bound IgG below a critical threshold necessary to support autoagglutination and complement activation.

WBEx may also have removed plasma factor(s) capable of exacerbating hemolysis and premature eryptosis analogous to that described in hyperhemolysis syndrome [30]. One possible candidate is methemoglobin, a potent oxidant, which can precipitate hemolysis by intra- and extra-erythrocytic mechanisms. Intracellular methemoglobin associates with Band 3 and cytoskeleton, leading to Heinz body formation, oxidative damage and altered RBC deformability [29]. More recent studies show that extracellular methemoglobin also leads to oxidative damage to the RBC membrane with increases in $\mathrm{RBC}$ fragility, aggregability and phosphatidylserine exposure. Because methemoglobin is rapidly generated from free Hgb following RBC lysis, it is hypothesized that free methemoglobin may synergize and amplify ongoing hemolysis in malaria and other hemolytic disorders [31,32].

There are only a few published reports of WBEx in AIHA (Table 1). There are two prior reports in classic WAIHA, although the methods and replacement fluids used differ from classic manual WBEx. Garelli and colleagues described a single WBEx in a 40 year old man with severe post-infectious WAIHA [14]. This patient initially underwent a 2 unit manual RBC exchange, followed by automated plasmapheresis using a cocktail of WB, plasma and colloid for replacement fluids. Like our patient, the patient had rapid improvement within 24 hours. A second case was reported in a recent abstract and describes the use of concurrent $2 \mathrm{~L}$ WB phlebotomy and reconstituted RBC transfusion using two synchronized infusion pumps in a woman with severe refractory WAIHA and chronic lymphocytic leukemia (CLL) [15]. Unlike our case, RBC was often reconstituted with saline, albumin, plasmalyte or plasma. The patient was successfully supported over 9 days with stabilization and rise in Hgb and a decrease in autoantibody levels (IAT, 2+ to $\mathrm{w}+$ ).

There are also three reports using WBEx in IgM-mediated AIHA (Table 1). John Hopkins reported the use of multiple WBEx in two pediatric cases with IgM-mediated WAIHA [4,5]. One child was an 11 month old girl with severe combined immunodeficiency (SCID) who underwent a related haploidentical bone marrow transplantation complicated by a severe WAIHA on day 2 [5]. The second patient was a 9 year old with Evan's syndrome [4]. Both patients received aggressive immunosuppression and twice daily WBEx with ongoing hemolysis, hemagglutination, multiorgan injury and death within weeks. A third case was reported by Schonitzer, who reported a fatal case of an antiPr cold AIHA secondary to Mycoplasma infection [16]. This patient received multiple automated WBEx totaling 41 units of blood without improvement and death 5 days after presentation. The inability of WBEx and aggressive immunosuppression to moderate hemolysis in these three cases is testimony to the unique severity of AIHA due to warm-acting, high-affinity IgM autoantibodies [2,3,33,34].

The severity of our patient's presentation did raise concerns of a warm IgM component to his AIHA. Like an IgM-WAIHA, the patient presented with an unusually severe, unresponsive AIHA, with in vitro and in vivo $\mathrm{RBC}$ autoagglutination, worsening hypoxia and impaired peripheral circulation with mottling and cyanosis. However, the patient's serologic workup did not fit a classic cold or warm IgM autoantibody. The patient's antibody was optimally reactive at $37^{\circ} \mathrm{C}$ and anti-IgG, with no enhancement at room temperature. In addition, the DAT with warm washed RBC was overwhelming IgG (4+) whereas IgMmediated AIHA is characterized by strong complement binding [2,3]. The transient C3 positivity observed immediately post-splenectomy may reflect the critical role of splenic macrophages in clearing C3coated RBC [2]. Finally, there was no change in antibody strength or reactivity after treatment of plasma with 2-ME, thus excluding an IgM autoantibody [3,17].

There are anecdotal case reports of plasmapheresis in patients with severe refractory AIHA. Although a decrease in autoantibody can often be documented, clinical efficacy is highly variable with many studies reporting no apparent decrease in hemolysis or transfusion frequency $[11,35]$. Plasmapheresis may be particularly ineffective in classic IgGmediated WAIHA since IgG has limited intravascular distribution (40-45\%), finite removal by apheresis and rapid post-procedure rebound [36]. In patients with severe WAIHA, it may be extremely difficult to significantly reduce autoantibody levels due to high titers and biosynthetic rates. Not surprisingly, reports of plasmapheresis successfully moderating hemolysis involved multiple apheresis procedures in conjunction with aggressive immunosuppression $[12,13]$. Furthermore, the circulating free autoantibody present in plasma represents only a small fraction of the total intravascular autoantibodymost of which has already bound and sensitized RBC. At present, plasmapheresis for WAIHA is considered a category III indication by the American Society of Apheresis [37].

In summary, we describe the successful use of manual WBEx in life-threatening WAIHA. A single WBEx was well tolerated; with acceptable decreases in plasma free Hgb, triglycerides, methemoglobin, and resolution of RBC autoagglutination. The improved oxygenation and perfusion observed post-WBEx may correspond to correction of the $\mathrm{O}_{2}$ dissociation curve, blood viscosity and peripheral vasodilation due to methemoglobin, $\mathrm{RBC}$ agglutinates and free $\mathrm{Hgb}$, respectively [29]. WBEx may also have quieted hemolysis through removal of heavily sensitized RBC (IgG+, C3+), in vivo alloadsorption of autoantibody and circulating methemoglobin. Advantages of manual WBEx over plasmapheresis are safety, concurrent removal of RBC and plasma; ability to provide aggressive isovolemic RBC transfusion support; and lack of expensive equipment and skilled personnel. Disadvantages are the time and labor, especially in adults with large blood volumes. Based on the literature, WBEx may be more beneficial in IgG-mediated WAIHA.

\section{Acknowledgment}

The authors would like to acknowledge and thank the blood bank and ICU nursing staff for their indispensable hard work in caring for this patient. Thanks to Louanne Dake MT (SBB), Immunohematology Reference Laboratory, for serologic evaluation of this patient. Thanks to Dr. Jennifer Hummel and Suzanne Butch MT (SBB) for photographs and Elizabeth Walker for help with figures. Finally, the author (LC) thanks Dr. Karen King, John Hopkins University for useful discussion and consultation regarding this case.

\section{References}


Citation: Cooling L, Boxer G, Simon R (2013) Life-Threatening Autoimmune Hemolytic Anemia Treated with Manual Whole Blood Exchange with Rapid Clinical Improvement. J Blood Disorders Transf 4: 163. doi: 10.4172/2155-9864.1000163

1. Barros MM, Blajchman MA, Bordin JO (2010) Warm autoimmune hemolytic anemia: recent progress in understanding the immunobiology and the treatment. Transfus Med Rev 24: 195-210.

2. Kelton JG, Chan HW, Heddle NM, Whittaker S (2003) Acquired hemolytic anemia. In: Wickramasinghe SN, McCullough J (Eds.), Blood and Bone Marrow Pathology, Churchill Livingston, Elsevier Science Limited, Edinburgh, Scotland pp 185-202.

3. Arndt PA, Leger RM, Garratty G (2009) Serologic findings in autoimmune hemolytic anemia associated with immunoglobulin $\mathrm{M}$ warm autoantibodies. Transfusion 49: 235-242

4. Friedmann AM, King KE, Shirey RS, Resar LM, Casella JF (1998) Fata autoimmune hemolytic anemia in a child due to warm-reactive immunoglobulin M antibody. J Pediatr Hematol Oncol 20: 502-505.

5. Nowak-Wegrzyn A, King KE, Shirey RS, Chen AR, McDonough C, et al. (2001) Fatal warm autoimmune hemolytic anemia resulting from IgM autoagglutinins in an infant with severe combined immunodeficiency. J Pediatr Hematol Onco 23: $250-252$

6. Habibi B, Homberg JC, Schaison G, Salmon C (1974) Autoimmune hemolytic anemia in children. A review of 80 cases. Am J Med 56: 61-69.

7. Aladjidi N, Leverger G, Leblanc T, Picat MQ, Michel G, et al. (2011) New insights into childhood autoimmune hemolytic anemia: a French national observational study of 265 children. Haematologica 96: 655-663.

8. Gómez-Almaguer D, Solano-Genesta M, Tarín-Arzaga L, Herrera-Garza JL, Cantú-Rodríguez OG, et al. (2010) Low-dose rituximab and alemtuzumab combination therapy for patients with steroid-refractory autoimmune cytopenias. Blood 116: 4783-4785.

9. Rossignol J, Michallet AS, Oberic L, Picard M, Garon A, et al. (2011) Rituximabcyclophosphamide-dexamethasone combination in the management of autoimmune cytopenias associated with chronic lymphocytic leukemia. Leukemia 25: 473-478.

10. Shvidel L, Sigler E, Shtalrid M, Berrebi A (2006) Vincristine-loaded platele infusion for treatment of refractory autoimmune hemolytic anemia and chronic immune thrombocytopenia: rethinking old cures. Am J Hematol 81: 423-425.

11. McLeod BC (2007) Evidence based therapeutic apheresis in autoimmune and other hemolytic anemias. Curr Opin Hematol 14: 647-654.

12. Lucchini G, Masera N, Foti G, Assali G, Perseghin P, et al. (2009) A lifethreatening paediatric case of acute autoimmune haemolytic anaemia (AlHA) successfully cured by plasma-exchange and combined immunosuppressive treatment. Transf Apheresis Sci 40:115-118.

13. Aglieco F, Manickaratnam S, Bona R, Kaplan AA (2008) A case report of refractory warm autoimmune hemolytic anemia treated with plasmapheresis and rituximab. Ther Apher Dial 12: 185-189.

14. Garelli S, Montani F, Navassa G, Restelli G (1985) Exchange transfusion and plasma-exchange in acute autoimmune hemolytic anemia: report of one case. Haematologica 70: 166-170.

15. Becker J, Scranton K (2013) Semi-automating the whole blood exchanges. J Clin Apher 28: 116

16. Schönitzer D, Kilga-Nogler S, Trenkwalder B, Lisch H, Breier C, et al. (1985) [Autoimmune hemolysis caused by anti-Pr]. Infusionsther Klin Ernahr 12: 181-184.

17. Judd WJ (1994) Methods in Immunohematology (2ndEdn.), Durham NC Montgomery Scientific Publications.

18. Branch DR, Petz LD (1982) A new reagent (ZZAP) having multiple applications in immunohematology. Am J Clin Pathol 78: 161-167.

19. Marsh WL (1972) Scoring of hemagglutination reactions. Transfusion 12: 352-353.

20. Sotelo-Avila C, Brouillette RT Jr, Gould SD (1982) The hematocrit of reconstituted blood for exchange transfusion in newborn infants. J Pediatr 100 971-972.

21. Carson TH (2012) Standards for blood banks and transfusion services (28th Edn.), AABB Press, Bethseda MD, USA

Citation: Cooling L, Boxer G, Simon R (2013) Life-Threatening Autoimmune Hemolytic Anemia Treated with Manual Whole Blood Exchange with Rapid Clinical Improvement. J Blood Disorders Transf 4: 163. doi: 10.4172/21559864.1000163
22. Druml W, Grimm G, Laggner AN, Schneeweiss B, Lenz K (1991) Hyperlipidemia in acute hemolysis. Klin Wochenschr 69: 426-429.

23. Druml W, Laggner AN, Lenz K, Grimm G, Schneeweiss B (1991) Pancreatitis in acute hemolysis. Ann Hematol 63: 39-41.

24. Saruc M, Yuceyar H, Turkel N, Ozutemiz O, Tuzcuoglu I, et al. (2003) An experimental model of hemolysis-induced acute pancreatitis. Braz $\mathrm{J}$ Med Biol Res 36: 879-886.

25. Schaer DJ, Buehler PW, Alayash AI, Belcher JD, Vercellotti GM (2013) Hemolysis and free hemoglobin revisited: exploring hemoglobin and hemin scavengers as a novel class of therapeutic proteins. Blood 121: 1276-1284.

26. Borinstein SC, Xu M, Hawkins DS (2008) Methemoglobinemia and hemolytic anemia caused by rasburicase administration in a newly diagnosed child with Burkitt lymphoma/leukemia. Pediatr Blood Cancer 50: 189.

27. Bhat P, Sisler I, Collier AB 3rd (2008) Exchange transfusion as treatmen for rasburicase induced methemoglobinemia in a glucose-6-phosphate dehydrogenase deficient patient. Pediatr Blood Cancer 51: 568.

28. Browning LA, Kruse JA (2005) Hemolysis and methemoglobinemia secondary to rasburicase administration. Ann Pharmacother 39: 1932-1935.

29. Kenneth Kaushansky, Marshall Lichtman, Thomas Kipps, Josef Prchal, Ur Seligsohn, et al. (2010) Williams Hematology (8th Edn.), McGraw-Hill, New York, USA.

30. Chadebech P, Habibi A, Nzouakou R, Bachir D, Meunier-Costes N, et al. (2009) Delayed hemolytic transfusion reaction in sickle cell disease patients: evidence of an emerging syndrome with suicidal red blood cell death. Transfusion 49 : 1785-1792.

31. Balaji SN, Trivedi V (2012) Extracellular Methemoglobin Mediated Early ROS Spike Triggers Osmotic Fragility and RBC Destruction: An Insight into the Enhanced Hemolysis During Malaria. Indian J Clin Biochem 27: 178-185.

32. Balaji SN, Trivedi V (2013) Extracellular methemoglobin primes red blood cell aggregation in malaria: an in vitro mechanistic study. FEBS Lett 587: 350-357.

33. McCann EL, Shirey RS, Kickler TS, Ness PM (1992) IgM autoagglutinins in warm autoimmune hemolytic anemia: a poor prognostic feature. Acta Haematol 88: $120-125$

34. Garratty G, Arndt P, Domen R, Clarke A, Sutphen-Shaw D, et al. (1997) Severe autoimmune hemolytic anemia associated with IgM warm autoantibodies directed against determinants on or associated with glycophorin A. Vox Sang 72: $124-130$

35. Ruivard M, Tournilhac O, Montel S, Fouilhoux AC, Quainon F, et al. (2006) Plasma exchanges do not increase red blood cell transfusion efficiency in severe autoimmune hemolytic anemia: a retrospective case-control study. J Clin Apher 21: 202-206.

36. Weinstein R (2010) Basic principles of therapeutic blood exchange. In: McLeod BC, Szczepiorkowski ZM, Weinstein R, Winters JL, (Eds.), Apheresis: Principles and Practice (3rdedn.), AABB Press, Bethesda MD, USA, pp 269-293.

37. Schwartz J, Winters JL, Padmanabhan A, Balogun RA, Delaney M, et al. (2013) Guidelines on the use of therapeutic apheresis in clinical practice-evidencebased approach from the Writing Committee of the American Society for Apheresis: the sixth special issue. J Clin Apher 28: 145-284. 